\title{
Examining measurement isomorphism of multilevel constructs: The case of political trust
}

Anna Ruelens - Research Institute for Work and Society, University of Leuven (Belgium)

Bart Meuleman - Centre for Sociological Research, University of Leuven (Belgium)

Ides Nicaise - Research Institute for Work and Society, University of Leuven (Belgium)

\section{Accepted for publication in Social Indicators Research}

The final authenticated version is available online at: https://doi.org/10.1007/s11205-017-1799-6

Corresponding author:

Anna Ruelens

Research Institute for Work and Society, University of Leuven, Belgium

Parkstraat 47 box 5300

B-3000 Leuven

Belgium

Funding: This research was part of the Re-InVEST project (Rebuilding an Inclusive, Value-based Europe of Solidarity and Trust through Social Investments), funded by the European Union's Horizon 2020 research and innovation programme under Grant Agreement No 649447.

Conflict of interest: The authors declare that they have no conflict of interest. 


\title{
Examining measurement isomorphism of multilevel constructs: The case of political trust
}

\begin{abstract}
There is a growing recognition of the importance of testing for measurement equivalence when comparing latent constructs - such as political trust - across countries and over time. Indeed, equivalence of measurements across countries and time points is a precondition for making meaningful and valid comparisons of means, scores, and relationships between constructs. Until recently, most efforts in this area of comparative research have focused on establishing measurement equivalence across groups, such as countries or cultural regions. In contrast, scarce attention has been paid to examining the measurement equivalence of constructs across levels of data. Rather, most empirical studies that use cross-national survey data assume that the factorial structure of a given construct is isomorphic, in other words, similar in measurement and meaning at the individual and country level. This assumption is not always justified, as the dimensions found at the individual level of data do not always generalize to the country level. In such cases, the results and substantive conclusions based on the assumption of measurement isomorphism may be misleading. In this article, we emphasize the importance of examining measurement isomorphism when working with crossnational survey data and describe a testing procedure using multilevel confirmatory factor analysis. We illustrate the procedure by examining cross-level equivalence of the political trust scale included in the European Social Survey (2008-2009). The results of the analysis indicate that the structure of political trust factor can be considered configurally isomorphic across individual and country levels. In addition, the measurement scale across the two levels is found to be partially isomorphic. These findings could be regarded as an encouraging result for the applied researchers who use the aggregated individual scores of political trust. At the same time, we demonstrate that measurement isomorphism cannot be simply assumed and hence should be examined prior to analysis to ensure valid and meaningful results.
\end{abstract}

Key words: Political trust, European Social Survey, Measurement isomorphism, Measurement equivalence, Multilevel confirmatory factor analysis 


\section{Examining measurement isomorphism of multilevel constructs: The case of political trust}

\section{Introduction}

In recent years, scholars of comparative cross-national research have been paying increasing attention to the issue of measurement equivalence when comparing latent constructs (Meuleman et al. 2009; Van der Veld and Saris 2011, Davidov et al. 2014), such as political trust (Marien 2011, 2017; Poznyak et al. 2014; André 2014; Schneider 2016; Turper and Aarts 2017). Measurement equivalence refers to a specific aspect of the validity of instruments, namely to the question of whether or not "under different conditions of observing and studying phenomena, measurement operations yield measures of the same attribute" (Horn and McArdle 1992, p. 117). It is now well-recognized that establishing measurement equivalence of a construct is necessary before valid cross-country comparisons can be made (Davidov et al. 2014). Although the use of measurement equivalence testing has yet to become common practice in cross-national assessment of political trust attitudes, a number of recent studies have especially contributed to improving our understanding of comparability of political trust instruments across contexts. Using the political trust items included in the European Social Survey (ESS), Marien (2011) conducted a thorough investigation of cross-national comparability of the 11-point political trust scale. The equivalence tests reveal that political trust can be meaningfully discussed in all democratic countries included in the ESS, and can be used for crossnational comparison (Marien 2011). These findings have been confirmed in more recent work which extends the analyses to the data from Round 6 (2012) of the ESS (Marien 2017). Similarly, André (2014) in examining differences in political trust attitudes between migrant and native population in the European Union using the ESS data found support for metric equivalence of political trust scale while full scalar equivalence has not been achieved. More recently, Schneider (2016) using the Life in Transition Survey II (LITS II) conducted by the World Bank and European Bank for Reconstruction and Development, tested four different models of political trust in order to investigate the measurement equivalence of political trust scale across diverse regime types. Two of the four models reached partial scalar invariance making it possible to compare means of latent political trust factor across the subset of countries (André 2014). Measurement equivalence of political trust has been investigated not only across contexts but also across time periods. Using multigroup confirmatory factor analysis with the data from the American National Election Studies (ANES), Poznyak et al. (2014) established that the ANES political trust scale achieves partial measurement equivalence and is generalizable across survey waves. On the other hand, full measurement equivalence has not been reached as two of the scale items showed significant variation over time (Poznyak et al. 2014). In another longitudinal study, Turper and Aarts (2017) using the ESS data for the Netherlands have 
established partial scalar invariance of the political trust scale for the high and low political sophistication groups across five waves of survey data (2004 - 2012).

All the above-mentioned studies have highlighted the importance of testing for measurement equivalence between groups and time periods prior to inferring substantive conclusions about political trust from survey data. At the same time, the large cross-country survey data sets are characterized not only by the presence of multiple groups but also by the hierarchical structure of the survey data, with individual respondents nested within regions and countries. Given that much of the empirical comparative research in this area relies on the constructs measured using individual-level indicators to draw inferences at the country level, it is crucial that measurement equivalence is not only established across different groups but also across different levels of data. The question of whether the measurement of latent constructs is isomorphic - i.e. does not differ across levels (Fontaine 2008; Fontaine and Fischer 2011) - is relevant for several reasons.

First, the dimensionality of factor structure could differ across levels. For example, while a single level confirmatory factor analysis (CFA) may establish a two-factor solution, the results of the multilevel confirmatory factor analysis (MCFA) can find a two-factor solution at the individual level and only one factor at the country level. In this case, the assumption of cross-level equivalence does not hold and the conclusions derived from aggregated data may be misleading. Second, even if the same number of factors is present across levels, the factor loadings may not be equal across levels, meaning that the individual-level and the country-level constructs are measured on a different metric and these scales are not directly comparable. Finally, the issue of construct validity aside, by not exploring the factor structure of a construct across levels, the researcher runs the risk of overlooking relevant methodological and theoretical implications resulting from the use of hierarchical data.

Although the research on cross-level equivalence and measurement isomorphism has an established tradition dating back to the work of Cronbach (1976) and Härnqvist (1978), most empirical studies in comparative cross-national research use single-level factor analytic techniques to validate concepts, thereby assuming rather than testing that the factor solutions are isomorphic. One notable exception is a study by Fischer (2012) on isomorphism of value constructs using the 21 Portrait Value Survey items included in the ESS survey. Other exceptions originating in cross-cultural research include Van de Vijver and Watkins (2006), Byrne and Van de Vijver (2014), and Stankov and Saucier (2015). Given the general lack of attention to cross-level equivalence testing, Byrne and Van de Vijver (2014) have described the investigation of measurement equivalence across levels as "probably the most underrated topic in cross-cultural research methods" (p.170). Similarly, Puntscher et al. (2016) have raised the issue of cross-level equivalence in their discussion of the aggregated measure of social capital. The authors particularly note that the effects of the aggregation process on the interpretation of 
latent constructs across levels are only rarely considered in academic research (Puntscher et al. 2016; also see Van de Vijver and Poortinga 2002).

In this article, we address this shortcoming by investigating the presence of isomorphism in a well-validated and often-used instrument for measuring political trust included in the European Social Survey. This study uses Round 4 (2008-2009) of the ESS data in order to build on the measurement equivalence analyses of political trust provided in Marien (2011). By using this example, we also intend to demonstrate the differences in logic and procedure between testing for multigroup equivalence and measurement isomorphism.

Building on the literature on measurement isomorphism and on the recent work on cross-level equivalence, this article aims to make three distinct contributions to the cross-national measurement equivalence research, in general, and political trust literature, in particular. First, we discuss the notion of measurement isomorphism and elaborate on the problems of assuming the similarity of factor structure across levels without testing this assumption explicitly. Second, we suggest a generic test procedure for examining measurement isomorphism of latent constructs, using a multilevel confirmatory factor analytic (MCFA) approach (Hox et. al. 2010; Muthén 1991) within the framework of multilevel structural equation modeling (MLSEM) (Goldstein and McDonald 1988; Muthén 1990, 1994; Muthén and Satorra 1995; Hox et al. 2010; Cheung and Au 2005). Finally, we empirically examine the structure of a frequently used political trust scale and compare our estimates with the estimates from a single-level CFA approach. In so doing, the present study constitutes one of the first systematic examinations of measurement isomorphism of political trust construct.

The article proceeds as follows. In Section 2 we discuss the conceptual underpinnings of measurement isomorphism, couching this discussion within the context of measurement equivalence research. In Section 3, we describe and explain a procedure for examining measurement isomorphism within multilevel factor analytic framework. In Section 4, we apply the procedure to our empirical example of political trust and summarize the results of the analyses. Finally, Section 5 provides the discussion and conclusion of the present work.

\section{Cross-group measurement equivalence vs. cross-level measurement isomorphism}

\subsection{Measurement equivalence across groups}

The issue of comparability of measurements across different contexts has become especially prominent with the wide availability of cross-national survey data sets, such as the European Social Survey (ESS), the World Value Survey (WVS), or the International Social Survey Programme (ISSP). Due to the fact that comparative surveys are administered across different countries and regions, the 
comparability of the measures can be compromised. For instance, cultural contexts and language differences can impact how respondents understand and evaluate a particular concept of interest, such as social capital, political trust, or well-being. Testing for measurement equivalence of an instrument ensures that the instrument measures the same construct across various contexts. In other words, when the same set of questions indicating a particular construct is asked in different contexts, the meaning of this construct as well as the scale on which the construct is measured, are sufficiently similar (Davidov et al. 2014).

A number of different statistical methods have been used to assess measurement equivalence of a construct. These include multigroup confirmatory factor analysis (MGCFA), multilevel mixture item response theory (IRT), and latent class analysis. More recently, multilevel structural equation modeling (MLSEM) has been suggested as an alternative to more conventional techniques for assessing measurement equivalence across contexts (Jak 2017; Davidov et al. 2012). Within the MGCFA tradition - arguably the most frequently used in cross-national research - three levels of measurement equivalence are distinguished, namely configural, metric and scalar equivalence (Steenkamp and Baumgartner, 1998; Vandenberg and Lance, 2000). ${ }^{1}$ Configural equivalence represents the lowest level of equivalence and refers to the similarity of the patterns of relations between latent construct and indicators across different groups. When configural equivalence is reached, the concept can be meaningfully discussed in each of the relevant contexts (i.e., no construct bias is present (Van de Vijver 1998). Metric equivalence refers to the equality of factor loadings across groups, and ensures the similarity of scale intervals across contexts. To provide an example, metric equivalence means that a one-unit increase in a latent construct, such as political trust, has the same meaning in all countries under study (Marien 2017). Finally, scalar equivalence presupposes that not only factor loadings, but also item intercepts are equal across groups. As such, scalar equivalence establishes whether the origin of the scale used to measure the construct is identical across different contexts. When scalar equivalence is established, one can compare mean levels of a construct across contexts (Meredith 1993; Vandenberg and Lance 2000).

\subsection{Isomorphism: Measurement equivalence across levels}

Although the importance of assessing measurement equivalence across groups have been well acknowledged, scarce attention has been given to examining measurement equivalence across levels of survey data. Cross-group equivalence and cross-level equivalence are conceptually and operationally two different issues, with diverging consequences for comparability. The former refers to the structural equivalence of a construct at the individual level in each of the cultural groups, and is required to

\footnotetext{
${ }^{1}$ Metric and scalar invariance are also referred in the literature as week and strong factorial invariance, respectively.
} 
compare measurements from one group to the next. The latter, conversely, relates to similarity in measurement models between the individual and the group levels of analysis and is a necessary (but not sufficient) condition for drawing conclusions that involve data at multiple levels (Fontaine 2008). Up to date, only a limited number of studies have examined cross-level equivalence of latent constructs in the fields of cross-cultural studies (Cheung et al. 2006; Fontaine and Fischer 2011; Byrne and Van de Vijver 2014), leadership studies (Dyer et al. 2005), organizational research (Tay et al. 2014), and educational research (D’Haenens et al. 2010; Schweig 2014).

Building on previous work by Van de Vijver, Van Hemert and Poortinga (2008) and Fontaine and Fischer (2011), we use the notion of 'measurement isomorphism' to refer to the similarity of meaning of a latent construct at different levels of analysis. Concretely, measurement isomorphism is assessed by testing whether the associations between indicators and latent constructs at the individual level (thus within countries) are similar with the associations between indicators and latent constructs at the country level (i.e., between countries). When a construct is found to be non-isomorphic, this implies that the meaning of the construct or its mapping onto a measurement scale is not identical at the individual and country level of analysis. As a consequence, the comparison of the latent construct between the two levels can lack validity. Probably one of the established examples of nonisomorphism comes from the cross-cultural research on human values (Schwartz 1992, 1994, 2006). In his investigation of value domains, Schwartz (1994) observed a different number of value dimensions and different relationships between value indicators, such as "social power and "humble", at the individual and at the cultural level. These findings clearly questions the validity of comparing cultural groups on the basis of individual-level scales alone (Fontaine and Fischer 2011). The issue of isomorphism is of particular relevance for the often-used multilevel regression models that decompose the variation of a concept measured at the individual level (such as political trust) into a within-group and between-group component. In doing so, such models assume similarity in the measurement of the within- and between-group variation. Clearly, single-level factor analysis provides insufficient empirical basis to warrant such comparisons, and cannot rule out the risk of committing an "aggregation error" (i.e., the incorrect generalization of the lower-level measurement characteristics to a higher level of analysis).

Similar to the hierarchically ordered levels of measurement equivalence across groups (Steenkamp and Baumgartner 1998; Meridith 1993), one can distinguish several levels of measurement isomorphism, although the exact number of levels is still debated (Tay et al. 2014). Most scholars agree that in order to be considered isomorphic a latent construct has to be configurally similar, namely to have an identical number of factors at the individual and country levels. This level of correspondence is referred to as configural isomorphism, and it implies that the construct under study has, qualitatively speaking, the same meaning at the individual and the country level. In order to make quantitative comparisons, the latent construct has to be measured on an identical scale, which 
requires that factor loadings to be equal across levels (Dyer et al., 2005). This level of isomorphism is termed metric isomorphism (Tay et al. 2014). When metric isomorphism holds, an increase of one unit on the measurement scale has the same meaning at the individual level as at the group level (Tay et al. 2014; Mehta and Neale 2005). Metric isomorphism is a requirement for estimating a multilevel regression model, in which individual predictors can simultaneously affect within and between-group differences in the dependent variable. ${ }^{2}$

Recent developments in multilevel structural equation modeling (MLSEM) (Goldstein and McDonald 1988, Muthén 1990, 1994; Muthén and Satorra 1995; Hox 2010; Cheung and Au 2005) and particularly in multilevel CFA (MCFA) (Hox et al. 2010, Muthén 1991) make it possible to operationalize and test the levels of isomorphism empirically. Given that most of the work in crossnational research utilizes two levels of analysis, namely the individual and the country level, in what follows, we focus on a two-level CFA model. It is important to note, however, that the two-level CFA model can also be extended to accommodate the analysis of a three-level structure (e.g. Jak 2014). The explanation below provides the necessary statistical guidance for understanding the procedure of cross-level equivalence testing using the two-level CFA model.

\section{Examining measurement isomorphism in the framework of multilevel CFA}

\subsection{The two-level CFA model}

A conventional single-level confirmatory factor analysis (CFA) aims to explain the covariance among $m$ variables that are observed across $i$ individuals $\left(y_{i}\right)$ in terms of $n$ latent variables $\left(\eta_{i}\right)$. The residuals $\left(\varepsilon_{i}\right)$ represent all unspecified sources of variability. Thus, the basic measurement model (Bollen 1989) can be specified as:

$$
\mathbf{y}_{i}=\tau+\Lambda \eta_{\mathbf{i}}+\varepsilon_{\mathbf{i}}
$$

where $\mathbf{y}_{\boldsymbol{i}}$ is a vector containing the observed score for individual $i, \boldsymbol{\eta}_{\mathbf{i}}$ contains the latent variable scores, and $\boldsymbol{\varepsilon}_{\mathbf{i}}$ is the residual or the error term for the $m$ th observed variable. The factor loadings $\boldsymbol{\Lambda}$ reflect the magnitude of change in the observed variables for unit of change in (one of) the latent variables, and $\boldsymbol{\tau}$ refers to the item intercepts (i.e. the expected score on observed variable $y$ when latent variable $\eta$ equals zero). This factor model implies the following structure of covariances $(\boldsymbol{\Sigma})$ and means $(\boldsymbol{\mu})$ for the observed variables:

$$
\boldsymbol{\Sigma}=\boldsymbol{\Lambda} \Psi \boldsymbol{\Lambda}^{\prime}+\boldsymbol{\Theta}(2)
$$

\footnotetext{
${ }^{2}$ In analogy to cross-group equivalence, one might also try to define the notion of scalar isomorphism. Such a notion is meaningless, however, since the intercept estimates only appear at the between level (by consequence, they cannot be set equal across levels).
} 


$$
\boldsymbol{\mu}=\boldsymbol{\tau}+\boldsymbol{\Lambda} \kappa(3)
$$

where $\boldsymbol{\Psi}$ is $n$ by $n$ matrix containing the latent variable (co)variances, $\boldsymbol{\Theta}$ is an $m$ by $m$ diagonal matrix containing residual variances, and $\boldsymbol{\kappa}$ is a vector with the latent means.

The two-level factor takes into account that individuals $i$ are not independent, but instead clustered in groups $j$. By consequence, separate measurement models for the individual-level and the group-level can be distinguished:

$$
\begin{gathered}
\mathbf{y}_{\mathbf{i j}}=\tau_{\mathbf{j}}+\Lambda_{\mathrm{W}} \boldsymbol{\eta}_{\mathrm{Wij}}+\boldsymbol{\varepsilon}_{\mathrm{Wij}}(4) \\
\tau_{\mathbf{j}}=\tau+\Lambda_{\mathrm{B}} \boldsymbol{\eta}_{\mathrm{Bj}}+\boldsymbol{\varepsilon}_{\mathrm{Bj}}(5)
\end{gathered}
$$

In equations 4 and 5, subscripts $\mathrm{w}$ and B refer to 'within' and 'between' levels, respectively. Equation (4) describes the within-group variation in observed variables $y_{i j}$ as a function of within-level latent variables $\eta_{W}$. Importantly, the within model includes random intercepts $\tau_{j}$ that vary over clusters $j$ and thus capture the group-level variation. Between-level equation (5) models this between-group variation as a function of between-level latent variables $\eta_{B}$.

By substituting equation (5) into (4), we obtain the full two-level CFA model (Hox et al. 2010):

$$
y_{i j}=\tau+\Lambda_{W} \eta_{W i j}+\Lambda_{B} \eta_{B j}+\varepsilon_{W i j}+\varepsilon_{B j}(6)
$$

where $\boldsymbol{\tau}$ is the vector of group level means, $\Lambda_{\mathbf{W}}$ is the matrix with factor loadings at the within level and $\boldsymbol{\Lambda}_{\mathbf{B}}$ is the between-level factor loading matrix. The terms $\boldsymbol{\varepsilon}_{\mathbf{W i j}}$ and $\boldsymbol{\varepsilon}_{\mathbf{B j}}$ represent the residuals at the within and the between level respectively. In the situation of one common factor at the within and between levels, a path diagram reflecting the above specifications is shown in Figure 1.

Figure 1 about here

Fig. 1 Path diagram of a two-level factor model. The path diagram is drawn using the conventions used by Muthén and Muthén (2004).

This two-level factor decomposes the total covariance matrix $\boldsymbol{\Sigma}$ as the sum of the within level $\left(\boldsymbol{\Sigma}_{\mathbf{W}}\right)$ and the between level covariance matrices ( $\left.\boldsymbol{\Sigma}_{\mathbf{B}}\right)$ (Muthén 1990; Rabe-Hesketh et al. 2004). In the case of individuals nested within countries, pooled-within covariance matrix $\boldsymbol{\Sigma}_{\mathbf{W}}$ describes how variables covary at the individual level within countries, while between-level covariance matrix $\boldsymbol{\Sigma}_{\mathbf{B}}$ contains the (co)variances at the country level. 
The within covariance matrix $\boldsymbol{\Sigma}_{\mathbf{W}}$ and the between covariance matrix $\boldsymbol{\Sigma}_{\mathbf{B}}$ can be reproduced by the two-level factor model, as follows:

$$
\begin{aligned}
& \boldsymbol{\Sigma}_{\mathrm{B}}=\Lambda_{\mathrm{B}} \Psi_{\mathrm{B}} \Lambda_{\mathrm{B}}^{\prime}+\boldsymbol{\Theta}_{\mathrm{B}} \\
& \boldsymbol{\Sigma}_{\mathrm{W}}=\Lambda_{\mathrm{W}} \Psi_{\mathrm{W}} \Lambda_{\mathrm{W}}^{\prime}+\boldsymbol{\Theta}_{\mathrm{W}}
\end{aligned}
$$

where $\boldsymbol{\Psi}_{\mathbf{B}}$ and $\boldsymbol{\Psi}_{\mathbf{W}}$ denote variance-covariance matrices of the latent factors at the between and within level, respectively, $\boldsymbol{\Theta}_{\mathbf{B}}$ and $\boldsymbol{\Theta}_{\mathbf{W}}$ are diagonal matrices with between- and within-level residual variances, and $\Lambda_{B}$ and $\Lambda_{W}$ contain the factor loadings at the between and the within levels.

The two-level CFA framework can be used to test the assumptions of cross-level isomorphism. First, when dimensionality of factor structures is identical across levels, configural isomorphism is considered to hold. This implies that the items exhibit the same configuration of factor loadings between the latent construct and the indicators at the individual and at the country level of analysis. When configural isomorphism is achieved, the latent construct can be meaningfully discussed at the individual as well as at the country level. Configural isomorphism can be tested by specifying identical measurement models for the within and between levels (without imposing constraints on the measurement parameters):

$$
\mathrm{H}_{0 \Lambda}: \text { pattern } \Lambda_{\mathbf{W}}=\text { pattern } \Lambda_{\mathbf{B}}(9)
$$

As mentioned above, however, configural isomorphism does not yet guarantee cross-level score comparability. Even if the dimensionality of factor structures is identical across levels, the scaling of the within and between latent variables will be different if the values of $\boldsymbol{\Lambda}_{\mathbf{W}}$ and $\boldsymbol{\Lambda}_{\mathrm{B}}$ diverge. In other words, metric isomorphism requires equal factor loadings across levels in addition to equal configural structure of the common factors. This level of cross-level equivalence can be assessed by testing the null hypotheses that denotes identical factor loadings across levels (Mehta and Neale 2005; Jak 2013; Muthén and Asparouhov 2013):

$$
\mathrm{H}_{0 \Lambda}: \Lambda_{\mathrm{W}}=\Lambda_{\mathrm{B}}
$$

If the equality of factor loadings across levels is established, the common factors have the same metric across levels (Muthén 1990; Rabe-Hesketh et al. 2004; Mehta and Neale 2005; Jak et al. 2014b), which, in turn, allows for the direct comparison of factors at the individual and the country-levels. This is a necessary condition for the valid application of multilevel regression models that rely on variance decomposition.

The following section outlines the analytical procedure for examining cross-level equivalence within the framework of MCFA. 


\subsection{Analytical procedure}

\subsubsection{A step-wise procedure for examining cross-level equivalence with multilevel CFA}

The suggested procedure presents a modification of the strategies outlined in Muthén (1994), Hox et al. (2010), and, more recently, in Jak et al. (2014) and consists of four main steps. Given that multilevel CFA approach is only appropriate when there is variance and covariance at the within as well as at the between level, in Step 1 it is necessary to calculate the intraclass correlation coefficient (ICC) of the indicators. The ICC summarizes the percentage of variance existing between higher-level units and can be viewed as an indicator of data dependency (Snijders and Bosker 1999). The statistical significance of the between-level variance and covariance can be tested by specifying a saturated model (i.e. a model with 0 degrees of freedom in which all variables are correlated with each other) for the within-level covariance matrix $\Sigma_{\mathrm{W}}$ while fitting a null model (i.e. a model where all variances and covariances are restricted to be zero: $\Sigma_{\mathrm{B}}=0$ ), and independence model ( $\Sigma_{\mathrm{B}}$ is diagonal) to the between-level covariance matrix (Hox et al. 2010; Muthén 1994, Jak et al. 2014). If the null model and the independence model do not fit the data, one can conclude that there is significant variance and covariance at the between-level.

In Step 2, one focuses on the individual-level measurement and estimates a measurement model at the within level, while specifying a saturated model at the between level (see above, a between model with 0 degrees of freedom, so that, by definition, there is no misfit at the between-level). Once a well-fitting model for the within level is found, one can proceed to Step 3 and test for configural isomorphism between levels. In this step, the aim is to establish whether the dimensionality of the within-level structure is similar or identical to the dimensionality of the between-level structure. This can be tested by specifying an identical model, i.e. a model with the same configuration of factor loadings at both levels. When configural isomorphism is established, one can proceed to Step 4 and test for metric isomorphism by estimating the model with equal factor loadings across levels. In Section 4, we demonstrate these steps by following the above procedure. The Mplus code is included in the supplementary material.

\subsubsection{Estimation}

All the analyses for this study were conducted with Mplus, version 7.4 (Muthén and Muthén 2012). For model estimation, we employ maximum likelihood estimation with robust standard errors (MLR) implemented in Mplus. The MLR is a commonly used estimator for MLSEM and MCFA and it is often used for continuous variables without assuming normality, while yielding a robust $\chi^{2}$ - test statistic (Kaplan et al., 2009). Given that one of the challenges of MCFA with cross-country data is the small number of units at the country-level (Meuleman and Billiet 2009), we re-estimate the final 
model with a Bayesian estimator (MCMC sampling) and then compare the parameter estimates and standard errors of the models estimated with MLR to those obtained with Bayesian estimation. The Bayesian estimator was shown to perform better when the sample size at the group level is small (Hox et al. 2012) and thus can serve as a robustness check for the estimates and standard errors for the models estimated with MLR. As such, performing a sensitivity analysis using Bayesian estimation is of crucial importance when using MLSEM with small sample sizes at the country-level, and is an integral part of our analytical strategy to test isomorphism in cross-national data.

The basic idea of Bayesian statistics is that prior knowledge (i.e. the prior distribution) is combined with the observed evidence (in the form of the likelihood of data given a set of parameters) to produce a posterior distribution of parameter estimates. This posterior distribution expresses the amount of uncertainty regarding the parameters that still exists after having observed the data. In the case of complex models such as MCFA, the posterior distribution can often not be solved analytically. Therefore, the posterior distribution is usually simulated using Monte Carlo Markov Chains (MCMC). The posterior distributions (and the derived 95\% credibility intervals) are obtained by means of an iterative procedure that estimates parameters repeatedly. As such, Bayesian estimation does not make assumptions about the distribution of test statistics, and is not dependent on large-sample theory (Kaplan and Depaoli 2012; van de Schoot and Depaoli 2014).

\subsubsection{Evaluation of fit}

For model evaluation, we suggest to proceed as follows. Since $\chi^{2}$ is known to reject reasonably specified models as a result of large sample sizes at the individual level, one can consult four additional indices when evaluating model quality estimated with MLR. These include: the TuckerLewis index (TLI), the comparative fit index (CFI), the root mean square error of approximation (RMSEA), and the standardized root mean square residual at the between $\left(\mathrm{SRMR}_{\mathrm{B}}\right)$ and the within levels $\left(\mathrm{SRMR}_{\mathrm{W}}\right)$. For the purposes of testing for isomorphism, it is especially important to examine SRMRb given that this measure evaluates the model fit for the between-level. Values of TLI and CFI at 0.95 or above indicate reasonably good fit (Hu and Bentler 1999). RMSEA/SRMR values smaller than 0.05 indicate close fit, while the values between 0.05 and 0.08 are deemed acceptable (Browne and Cudeck 1992). In this study, we use a cut-off value for RMSEA close to 0.06, as recommended by $\mathrm{Hu}$ and Bentler (1999).

For models estimated using Bayesian estimator, the fit measures are limited. While at present some alternatives exist for evaluating the fit of single-level CFA models, such as the Bayesian root mean square error of approximation (BRMSEA) (Hoofs et al. 2017), this measure is not available for assessing the fit of multilevel CFA models. Further, the posterior predictive $p$ value ( $p p p)$ used in Bayesian CFA is based on $\chi^{2}$ and therefore tends to reject reasonable model fit within large samples 
(Hoofs et al. 2017). Given this lack of fit measures for evaluating multilevel CFA models estimated with Bayesian estimator, we recommend: 1) using BRMSEA to assess a single-level CFA structure in order to ensure a good model fit at the within level; and 2) examining the autocorrelation plots provided with Mplus output to confirm that the estimated models have achieved convergence.

\section{Empirical Illustration: Examining isomorphism of political trust scale}

\subsection{Data}

We illustrate the proposed testing procedure by assessing measurement isomorphism of "political trust' factor using five items included in Round 4 of the European Social Survey data (2008). The ESS is a biennial cross-national European survey that is administered to probability-based samples from approximately thirty countries. While there are several advantages to using Round 4 of the ESS, including a large number of participating countries, we chose Round 4 so that a single-level one-factor measurement model of political trust provided in Marien (2011) can serve as a point of departure for our analysis. By relying on the data from the same round of the ESS data we are able: 1) to test whether the unidimensional model of political trust can be replicated at the country-level and 2) to demonstrate the differences between testing for multigroup equivalence and measurement isomorphism. ${ }^{3}$

The ESS asks respondents about their levels of trust in the national parliament, legal system, police, political parties, politicians, the European Parliament, and the United Nations. Given that most studies of political trust focus on domestic institutions, including the study of Marien $(2011,2017)$ we limit our analysis to the first five items. The responses are recorded on an eleven-point scale ranging from 0 ("having no trust at all") to 10 ("having complete trust"). Selected descriptive statistics for five items measuring the political trust latent variable, including the intraclass correlations, are presented in Table 1. Our sample includes 45,190 individuals nested in 28 countries. $^{4}$

\footnotetext{
${ }^{3}$ While a number of other cross-national survey instruments such as World Value Survey and Eurobarometer also collect data on political trust attitudes, the measurement scales of their trust items are less refined. More importantly, previous studies using the ESS indicators of political trust have demonstrated that the operationalization of trust in the ESS reveals a high level of validity and reliability (Marien 2011, 2017; André 2014).

${ }^{4}$ These countries include Belgium, Bulgaria, Croatia, Cyprus, the Czech Republic, Denmark, Estonia, Finland, France, Germany, Greece, Hungary, Ireland, Israel, Latvia, the Netherlands, Norway, Poland, Portugal, Romania, Slovakia, Slovenia, Spain, Sweden, Switzerland, Turkey, Ukraine, and the United Kingdom. Note: not all of the participating countries were included in the equivalence analyses in Marien (2011).
} 
Table 1 Descriptive statistics for political trust items in ESS Round 4 (2008-2009)

\begin{tabular}{lllll}
\hline Items & Mean & SD & Skew & ICC \\
\hline 1. Trust in National Parliament $^{5}$ & 4.27 & 2.66 & 0.01 & 0.241 \\
2. Trust in Legal System & 4.91 & 2.75 & -0.17 & 0.239 \\
3. Trust in Police & 5.60 & 2.74 & -0.44 & 0.231 \\
4. Trust in Politicians & 3.35 & 2.41 & 0.23 & 0.212 \\
5. Trust in Political Parties & 3.38 & 2.38 & 0.19 & 0.205 \\
\hline
\end{tabular}

\subsection{Measurement model of political trust}

Most of existing empirical research on trust in domestic political institutions tends to employ a unidimensional measurement of political trust (Zmerli and Newton 2008; Oskarsson 2010, Hooghe 2011; Marien 2011, 2017). There are, however, also those who find support for the multidimensional structure of political trust (Brug and Praag 2007; Rothstein and Stolle 2008, Allum et al. 2011). In particular, in some contexts, trust in the European Parliament and trust the United Nations are found to constitute a different dimension of political trust (Zmerli and Newton 2008). Similarly, at the level of domestic political institutions, some researchers distinguish between representative institutions, namely parliament, politicians and political parties, and implementing institutions, namely the legal system and the police (Marien 2011). These two sets of institutions have different responsibilities, such as representing the interests of the electorate, as in the case of political parties and politicians, or maintaining order, as in the case of the police (Rothstein and Stolle 2008). In addition, these domestic political institutions also face different expectations from their citizens. Rather than conceiving of implementing and representative institutions as two different dimensions of political trust, Marien (2011) proposes a one-dimensional model of political trust with two error correlations. Specifically, one error correlation is specified between Trust in Politicians and Trust in Political Parties and another error correlation is specified between Trust in Legal System and Trust in Police. In other words, these two pairs of indicators share some covariance that cannot be explained by the general concept of political trust. Given the theoretical arguments and the confirmatory nature of our study, we use this one-dimensional model with two error-correlations in our further analysis.

\subsection{Results}

\subsubsection{A stepwise test for the cross-level equivalence of political trust}

In Step 1, we inspect the ICCs of the indicators of political trust. The ICCs for the five items are as follows: Trust in National Parliament (0.241), Trust in Legal System (0.239), Trust in Police (0.231),

\footnotetext{
${ }^{5}$ For the purpose of clarity, we distinguish between 'Trust' as a variable (in upper case ex. 'Trust in Police') and trust as an attitude (in lower case).
} 
Trust in Politicians (0.212), Trust in Political Parties (0.205) (Table 1). The noticeable size of these coefficients suggests that there is considerable variability in the political trust items that exists at the country level. Put differently, about $20 \%$ of variance in each of the items is located at the group-level, while the remaining $80 \%$ consists of individual differences within countries. ICCs of this size provide sufficient evidence that multilevel approach is necessary.

Goodness-of-fit statistics for the estimated models are summarized in Table 2. To investigate whether this considerable amount of country-level variation is also statistically significant, we first estimate a model with a saturated model at the within level and a null model at the between level with no variances and covariances specified at the between level (Model 1, Step 1). Clearly, this model does not adequately fit the data $\left(\chi^{2}=23896.72, d f=15, \mathrm{p}<0.05\right.$, CFI $=0.000$, TLI $=-4.608$, RMSEA $\left.=0.188, \mathrm{SRMR}_{\mathrm{B}}=0.731\right)$, indicating that there is significant variance at the between level. An independence model (Model 2, Step 1) with a saturated model at the within level and no covariances specified at the between level is also rejected $\left(\chi^{2}=227.095, d f=10, \mathrm{p}<0.05\right.$, CFI $=0.962$, TLI $=$ 0.924, RMSEA $=0.022$, SRMR $\left._{\mathrm{B}}=0.731\right)$, which indicates that there exist significant interrelations between the indicators at the country level. In addition to a very poor fit at the between level with $\mathrm{SRMR}_{\mathrm{B}}$ greatly exceeding the accepted threshold of .08 , the independence model exhibited signs of inadmissible solution due to a non-positive definite first-order derivative product matrix. The difficulty with estimation of two level models is not uncommon given the small sample size at the country-level (Byrne 2013). We will address the issue of small sizes when discussing the results of the Bayesian estimation. Given the fit statistics of the null and the independence model, we conclude that a specification of multilevel model is necessary (Hox et al. 2010).

In Step 2, we define a measurement model at the within level (Model 3). In line with Marien (2011, 2017), we retain the one-factor model with the two error correlations at the within level while specifying a saturated model (i.e. a model in which all variables are correlated with each other) at the between level. A one-factor model fitted closely at the within level covariance matrix $\left(\chi^{2}=27.916, d f\right.$ $=3, \mathrm{p}<0.05, \mathrm{CFI}=0.996, \mathrm{TLI}=0.971, \mathrm{RMSEA}=0.014, \mathrm{SRMRw}=0.006)$.

In Step 3, we test for configural isomorphism by examining whether the structure of political trust factor is configurally similar at the within and between level. Model 4, the one-factor model with identical specification at both levels (i.e. one factor model with two error correlations) was estimated normally. ${ }^{6}$ Model 4 showed good fit to data at both levels: $\chi^{2}=50.364, d f=6, \mathrm{p}<0.05$, CFI $=0.992$, $\mathrm{TLI}=0.974$, RMSEA $=0.013$ ). Standardized root mean squared residual statistics for the between and within model was 0.001 and 0.006 , respectively, suggesting that the multilevel model did an adequate job in reproducing covariances at both levels. Substantively, these results provide evidence of

\footnotetext{
${ }^{6}$ In the output, Mplus issued an automatic warning message about the small number of clusters compared to a number of constrained parameters at the between level. Omitting the two error correlations at the between level has solved the issue.
} 
similarity of structure and, thus, configural isomorphism of political trust factor across levels. This has important repercussions for cross-national research into political trust: evidently, individual-level measures of trust can be meaningfully aggregated to the country level, and it is legitimate to use political trust as a country-level concept. The intraclass correlation coefficient (ICC) for Model 4, calculated as the ratio of the variance of the latent factor at the between-level to the total variation, is 0.29 indicating that the between-latent factor explains $29 \%$ of the total variance in the sample.

Finally, in Step 4, we constrain the loadings to be invariant across levels, in order to test for metric isomorphism and to determine whether the scale on which latent variable political trust is measured can be compared across levels. The invariance of factor loadings across levels "equates the scales of the latent common factor across levels, thus making latent factor variances directly comparable" (Mehta and Neale 2005). Model with the between-level and within-level factor loadings constrained to be equal (Model 5) fits the data fairly well: $\chi^{2}=79.825, d f=10, \mathrm{p}<0.05$, CFI $=$ $0.988, \mathrm{TLI}=0.975, \mathrm{RMSEA}=0.012$, albeit an increase in $\mathrm{SRMR}_{\mathrm{B}}$ to 0.053 . The inspection of the output suggests that two items representing trust in implementing institutions, namely Trust in Legal System and Trust in Police, serve as better indicators of political trust at the between level rather than at the within level. Indeed, the estimated model (Model 6a) with equal factor loadings for three indicators (Trust in National Parliament, Trust in Politicians, Trust in Political Parties) and free factor loadings for Trust in Legal System and Trust in Police (Model 6a), has resulted in decreased SRMR from 0.053 to 0.003 . Therefore, we can conclude that partial metric isomorphism holds across levels: Three indicators (namely Trust in National Parliament, Trust in Politicians, and Trust in Political Parties) have equal factor loadings across levels, and therefore the between factor can be interpreted as the aggregated version of the within level latent factor. In other words, an increase of one unit on the measurement scale of the within factor translates into the identical increase at the between level, which justifies the use of multilevel regression models explaining political trust by means of individual and contextual predictors. In contrast, the items reflecting trust in implementing institutions (Trust in Legal System and Trust in Police) cannot be considered equal across level, given that they are more indicative of political trust at the between level than at the within level.

Table 2 Summary of model fit statistics

\begin{tabular}{|c|c|c|c|c|c|c|c|}
\hline Model & Model description & $\chi^{2}(\mathrm{df})$ & CFI & TLI & RMSEA & $\mathrm{SRMR}_{\mathrm{W}}$ & $\mathrm{SRMR}_{\mathrm{B}}$ \\
\hline Model 1 & $\begin{array}{l}\text { Null model at the } \\
\text { between level }\end{array}$ & $23896.72(d f=15)$ & 0.000 & -4.608 & 0.188 & 0.056 & 0.731 \\
\hline Model 2 & $\begin{array}{l}\text { Independence model } \\
\text { at the between level }\end{array}$ & $227.095(d f=10)$ & 0.962 & 0.924 & 0.022 & 0.000 & 0.731 \\
\hline Model 3 & $\begin{array}{l}\text { Measurement model } \\
\text { at the within level }\end{array}$ & $27.916(d f=3)$ & 0.996 & 0.971 & 0.014 & 0.006 & 0.000 \\
\hline Model 4 & Configural model & $50.364(d f=6)$ & 0.992 & 0.974 & 0.013 & 0.006 & 0.001 \\
\hline Model 5 & Equal loadings & $79.825(d f=10)$ & 0.988 & 0.975 & 0.012 & 0.006 & 0.053 \\
\hline
\end{tabular}




$\begin{array}{llllllll}\text { Model 6a } & \begin{array}{l}\text { Free loadings for } \\ \text { Trust in Legal } \\ \text { System and Trust in }\end{array} & 65.057(d f=8) & 0.990 & 0.975 & 0.013 & 0.006 & 0.003 \\ & \begin{array}{l}\text { Police } \\ \text { Single-level CFA }\end{array} & 188.518(d f=3) & 0.999 & 0.996 & 0.037 & -\end{array}$

$\begin{array}{lllll}\text { Model } 7 \quad \text { Single-level CFA } & 188.518(d f=3) & 0.999 & 0.996 & 0.037\end{array}$

\subsubsection{Metric isomorphism: MLR vs. Bayesian parameter estimates}

Given that one of the main challenges of using MCFA with cross-national survey data is a small number of countries included in the higher levels sample which may result in model non-identification and the possible underestimation of standard errors, we re-estimate the final model (Model 6a) using Bayesian estimator available in Mplus 7.4, with default Mplus priors specified for factor loadings and variances. In multilevel literature, Bayesian estimation is suggested when the sample size at the grouplevel is small (Hox et al. 2012; Stegmueller 2013). The autocorrelation plots for Model 6b indicate that the model achieved convergence and the resulted estimates are very similar to the estimates of Model 6 estimated with MLR, supporting the robustness of our findings (Table 3).

Table 3 Unstandardized and standardized parameter estimates of Model 6 using MLR estimation and Model 7 Bayesian estimation

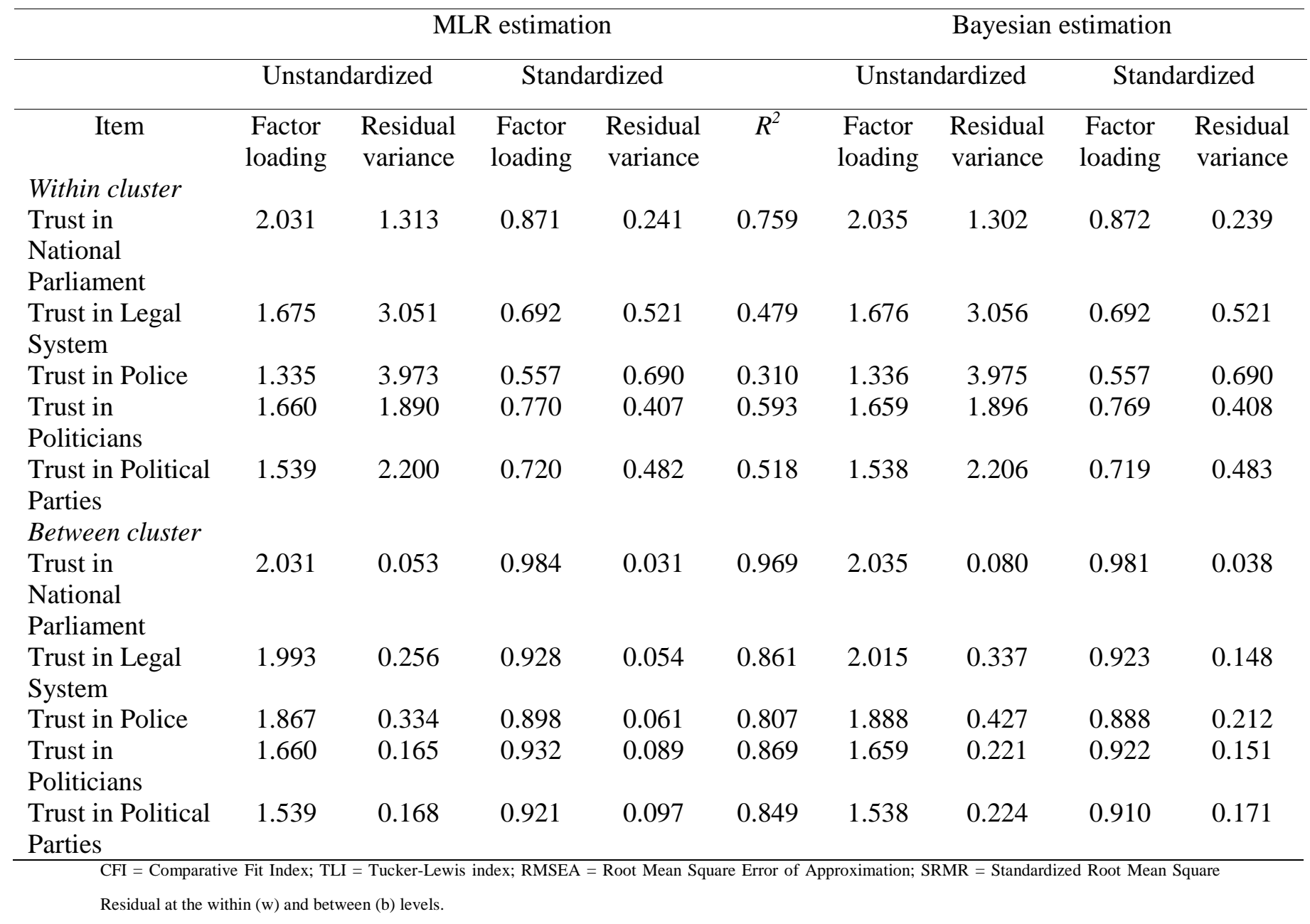


The proportion of variance explained by the latent political trust factor at the within level ranged from 0.310 to 0.759 . The corresponding proportions at the between level ranged from 0.807 to 0.969 . The lower proportion of variance explained at the within level suggests that after controlling for country variability in political trust, there remains considerable heterogeneity in the within-individual variability in political trust. Trust in Police item had the lowest $R^{2}$ both at the within and at the between level. It is generally acknowledged that trust in police may be a less important indicator of political trust (Marien 2011). This could explain the low $R^{2}$ for trust in police at both within- and between-clusters level. On the other hand, low $R^{2}$ for trust in police may indicate that there may exist some other factor influencing individual and country level variability in trust. Notable as well is that factor loadings at the between level were substantially higher than the comparable loadings at the within level. This reflects the notion that in the representation of multilevel measurement model, the items at the between level are affected by random measurement error to a much smaller extent only.

\subsubsection{Comparison of multilevel and single level CFA estimates}

Finally, to highlight the possible implications of using single-level factor analyses with multilevel data, we estimated Model 7 using the five political trust items. Using the covariance matrix of the pooled data, a single-level CFA was conducted using the MLR estimator. Model 7 showed a good fit to data: $\chi^{2}=188.518, d f=3, \mathrm{p}<0.05, \mathrm{CFI}=0.999$, TLI $=0.996$, RMSEA $=0.037$. However, when we compare the standardized parameter estimates from the MCFA model with the estimates resulting from the single-level CFA model (Table 4), we observe that the factor loadings are discrepant. While the pattern of the factor loadings are similar, the factor loadings retrieved here are situated in between the within and the between factor loadings from the two-level CFA. This illustrates that analyzing clustered data by means of single-level tools renders parameters that are a mixture of the within and between effects that is hard to interpret (Raudenbush and Bryk 2002). The factor loadings are drawn towards the within-level estimates, given that the statistical power at the lowest level $(\mathrm{N}=45,190)$ is much higher than at the country level $(\mathrm{N}=28)$. In this particular case, the discrepancy between standardized within and between effects is limited, due to the large similarities between the measurement models at both levels. When isomorphism is found to be absent, however, the discrepancies - and thus the extent to which single-level CFA is misleading - can only increase.

Table 4 Standardized parameter estimates of Model 6 using MLR estimation and the single-level CFA model (Model 7)

\begin{tabular}{llll}
\hline & $\begin{array}{l}\text { Single- } \\
\text { level CFA }\end{array}$ & Two-level CFA \\
\hline Item & & Within & Between \\
\hline $\begin{array}{l}\text { Trust in National } \\
\text { Parliament }\end{array}$ & 0.898 & 0.871 & 0.984 \\
Trust in Legal System & 0.748 & 0.692 & 0.928 \\
Trust in Police & 0.639 & 0.557 & 0.898 \\
Trust in Politicians & 0.800 & 0.770 & 0.932
\end{tabular}




\section{Discussion and conclusion}

When investigating constructs, empirical researchers often rely on multilevel data from cross-national surveys. Frequently, the indicators at the individual level are combined using single-level factor analytic techniques and consequently aggregated to the country-level to draw comparisons across countries. This paper argues that, for such comparisons to be valid, the structure of the factor(s) has to be equivalent or isomorphic across the individual and the country level of analysis. The aim of this study was (1) to emphasize the importance of testing for measurement isomorphism and (2) to illustrate the test procedure by assessing isomorphism of the well-established political trust scale.

While prior research has investigated cross-cultural measurement equivalence of the ESS indicators of political trust (Marien 2011, 2017; Poznyak et al. 2014; André 2014; Schneider 2016; Turper and Aarts 2017), the cross-level equivalence of the political trust scale has not been duly examined. Therefore, the present study constitutes the first systematic examination of cross-level measurement equivalence of the political trust scale using the fourth round of the ESS data. Specifically, in the empirical part of this study we were interested in investigating the question to what extent the political trust items that are included in the ESS data measure the same construct at the individual and country levels.

Based on multilevel confirmatory factor analyses, one overall factor of political trust was supported at both the individual and the country level. We can, therefore, conclude that the political trust latent factor measured using ESS (2008) indicators can be considered configurally isomorphic across the two levels. Substantively speaking, this configural isomorphism implies that the concept of political trust can be discussed meaningfully both at the individual and country level. By consequence, aggregation of the individual responses to the country-level for further analysis is valid. This is an encouraging result for the applied researchers who use the aggregated individual scores, as included in the ESS, to compare political trust in different countries. Furthermore, we also found support for partial metric isomorphism (that is, the equality of several factor loadings across levels). Accordingly, current findings indicate that the mapping of political trust factor onto a measurement scale is comparable across levels. In other words, not only what we mean by "trust in political institutions", but also the metric on which it is scaled can be generalized from the micro to the macro-(country) level. This (partial) metric isomorphism is a necessary condition for using analytical tools that compare trust scores at both levels numerically, such as multilevel regression models.

At the same time, our two-level factor analysis yields additional insights in the structure of micro- and macro-differences in the measurement of trust in implementing institutions. Two 
indicators, namely Trust in Legal System' and 'Trust in Police', act differently across the two levels. Substantively, this finding may indicate that that these two indicators are less indicative of a general factor of political trust, especially at the individual level. Given the theoretical arguments for distinguishing between representative and implementing institutions and the present results, this finding certainly deserves further investigation.

Furthermore, as our comparison of the results of single-level CFA and multilevel CFA demonstrates, while the pattern of factor loadings holds, the factor loadings and their standard errors from the single-level analysis differ from their counterparts from the two-level factor analysis. In cases in which isomorphism is established, we can conclude that the indicators act similar at different levels of analysis and thus the discrepancy between factor loadings may be of lesser importance for drawing conclusions on the basis of the single-level CFA. In the absence of isomorphism, however, we strongly recommend using multilevel approaches (MCFA or MLSEM) to properly model the relationships between indicators and latent variable (and other exogenous variables) to accurately reflect the relationships between them at multiple levels.

The results of this study have implications beyond the topic of political trust. As our conclusions indicate, cross-level equivalence of constructs across levels of aggregation has to be tested empirically rather than assumed. At present, most of the studies using multilevel data for comparative purposes implicitly assume the equivalence of structure and meaning of latent constructs across levels rather than explicitly testing for cross-level equivalence. As argued above, this assumption of isomorphism is problematic both from a statistical and a substantive perspective. Statistically, using single-level factor analytic techniques with multilevel data may violate the assumption of independence of observations and the assumption of homoscedasticity of residual variances. Substantively, a factor structure of a latent construct is assumed to be invariant across levels, while this assumption is not always justifiable. In our illustration the configural structure of political trust factor across levels was found to be identical. However, this need not always be the case. Instead, there can be significant differences in factorial structure across levels of analyses. In the absence of isomorphism, the aggregation of individual scores across countries can lead to misleading conclusions. For example, the number of dimensions can differ between levels and/or the meaning of constructs can be different across levels (Fontaine and Fisher 2011; Tay et al. 2014). In the situation when factorial structures are different at both levels, for example, when there are two factors present at the individual level and only one at the between level, if the factor analysis extract one factor, then the within level structure will be misrepresented (Zyphur et al. 2008). Furthermore, the importance of isomorphism is crucial not only for the research that makes use of the aggregated data but also for "any comparison of country means, derived from individual data, such as in t-tests or analyses of variance, assumes identity of meaning at the individual and country levels." (Van de Vijver and Watkins 2006). 
At the same time, researchers should not be afraid to find non-isomorphism when evaluating constructs under study. As we have argued above, the finding of non-isomorphism is theoretically as well as statistically interesting. If different factor structures or loadings are found at the individual and country level, this can constitute a theoretically interesting finding, as the work of Schwartz (1994, 2006) demonstrates. One of the main findings of Schwartz (1994) provides a clear example of nonisomorphism by revealing different dimensionality of human values across individual and cultural level. The conclusion of non-isomorphism indicates that the meaning and/or measurement of latent constructs differ between the individual and the country level. Such differences provide theoretically challenging puzzles, and stimulate new theoretical routes of investigating the sources of nonisomorphism that, in turn, would enrich both theoretical and empirical research. Concretely, when isomorphism is not supported, we advise to model both levels separately in order to explore what variables contribute to the differences in factor structure. Overall, our intention in this study was not only to caution about the consequences of non-isomorphism but, more importantly, to stimulate a more careful investigation into the internal structure of constructs when working with multilevel crossnational survey data. 


\section{References}

Allum, N., Read, S., \& Sturgis, P. (2011). Evaluating change in social and political trust in Europe. In E. Davidov, P. Schmidt, \& J. Billiet (Eds.), Cross-cultural analysis: Methods and applications (pp. 35-53). New York, London: Routledge.

André, S. (2014). Does trust mean the same for migrants and natives? Testing measurement models of political trust with multi-group confirmatory factor analysis. Social Indicators Research, 115(3), 963982.

Bollen, K. A. (1989). A new incremental fit index for general structural equation models. Sociological Methods \& Research, 17(3), 303-316.

Browne, M. W., \& Cudeck, R. (1992). Alternative ways of assessing model fit. Sociological Methods \& Research, 21(2), 230-258.

Byrne, B. M., 2013. Structural equation modeling with Mplus: Basic concepts, applications, and programming. Routledge.

Byrne, B. M., \& van de Vijver, F. J. (2014). Factorial structure of the Family Values Scale from a multilevel-multicultural perspective. International Journal of Testing, 14(2), 168-192.

Cheung, M. W. L., \& Au, K. (2005). Applications of multilevel structural equation modeling to crosscultural research. Structural Equation Modeling, 12(4), 598-619.

Cheung, M. W. L., Leung, K., \& Au, K. (2006). Evaluating multilevel models in cross-cultural research: An illustration with social axioms. Journal of Cross-Cultural Psychology, 37(5), 522-541.

Cronbach, L. J. (with Deken, J.E., \& Webb, N.). (1976). Research on Classrooms and Schools: Formulation of Questions, Design and Analysis. Occasional Paper of the Stanford Evaluation Consortium, Stanford University, Stanford, CA.

Davidov, E., Dülmer, H., Schlüter, E., Schmidt, P., \& Meuleman, B. (2012). Using a multilevel structural equation modeling approach to explain cross-cultural measurement noninvariance. Journal of Cross-Cultural Psychology, 43(4), 558-575.

Davidov, E., Meuleman, B., Cieciuch, J., Schmidt, P., \& Billiet, J. (2014). Measurement equivalence in cross-national research. Annual Review of Sociology, 40, 55-75.

D'haenens, E., Van Damme, J., \& Onghena, P. (2010). Multilevel exploratory factor analysis: illustrating its surplus value in educational effectiveness research. School Effectiveness and School Improvement, 21(2), 209-235.

Dyer, N. G., Hanges, P. J., \& Hall, R. J. (2005). Applying multilevel confirmatory factor analysis techniques to the study of leadership. The Leadership Quarterly, 16(1), 149-167.

Fischer, R. (2012). Value isomorphism in the European Social Survey: Exploration of meaning shifts in values across levels. Journal of Cross-Cultural Psychology, 43(6), 883-898.

Fontaine, J. (2008). Traditional and multilevel approaches in cross-cultural research: An integration of methodological frameworks. In F. J. Van de Vijver, D. A. Van Hemert, \& Y. H. Poortinga (Eds.), Multilevel analysis of individuals and cultures (65-92). Psychology Press.

Fontaine, J., \& Fischer, R. (2011). Data analytic approaches for investigating isomorphism between the individual-level and the cultural-level internal structure. In D. Matsumoto \& F. J. R. van de Vijver (Eds.) Cross-cultural research methods in psychology (pp. 273-298). New York: Cambridge University Press. 
Goldstein, H., \& McDonald, R. P. (1988). A general model for the analysis of multilevel data. Psychometrika, 53(4), 455-467.

Härnqvist, K. (1978). Primary Mental Abilities at collective and individual levels. Journal of Educational Psychology, 70(5), 706.

Hoofs, H., van de Schoot, R., Jansen, N. W., \& Kant, I. (2017). Evaluating Model Fit in Bayesian Confirmatory Factor Analysis With Large Samples: Simulation Study Introducing the BRMSEA. Educational and Psychological Measurement, doi: 10.1177/0013164417709314.

Hooghe, M., 2011. Why there is basically only one form of political trust. The British Journal of Politics \& International Relations, 13(2), pp.269-275.

Horn, J. L., \& McArdle, J. J. (1992). A practical and theoretical guide to measurement invariance in aging research. Experimental Aging Research, 18(3), 117-144.

Hox, J. J., Moerbeek, M., \& van de Schoot, R. (2010). Multilevel analysis: Techniques and applications. Routledge.

Hox, J., van de Schoot, R., \& Matthijsse, S. (2012). How few countries will do? Comparative survey analysis from a Bayesian perspective. Survey Research Methods, 6(2), 87-93.

Hu, L. T., \& Bentler, P. M. (1999). Cutoff criteria for fit indexes in covariance structure analysis: Conventional criteria versus new alternatives. Structural Equation Modeling: a Multidisciplinary Journal, 6(1), 1-55.

Jak, S. (2014a). Testing strong factorial invariance using three-level structural equation modeling. Frontiers in Psychology, 5, 745, doi:10.3389/fpsyg.2014.00745.

Jak, S. (2017). Testing and explaining differences in common and residual factors across many countries. Journal of Cross-Cultural Psychology, 48(1), 75-92.

Jak, S., Oort, F. J., \& Dolan, C. V. (2014b). Measurement bias in multilevel data. Structural Equation Modeling: A Multidisciplinary Journal, 21(1), 31-39.

Jak, S., Oort, F. J., \& Dolan, C. V. (2013). A test for cluster bias: Detecting violations of measurement invariance across clusters in multilevel data. Structural Equation Modeling: A Multidisciplinary Journal, 20(2), 265-282.

Kaplan, D., \& Depaoli, S. (2012). Bayesian structural equation modeling. In R. H. Hoyle (Ed.), Handbook of structural equation modelling (pp. 650-673). Guilford Press, New York, NY.

Kaplan, D., Kim, J. S., \& Kim, S. Y. (2009). Multilevel latent variable modeling: Current research and recent developments. In R.E. Millsap \& A. Maydeu-Olivares (Eds.), The SAGE handbook of quantitative methods in psychology (pp. 592-612). SAGE, Thousand Oaks, CA.

Marien, S. (2011). Measuring political trust across time and space. In M. Hooghe M. \& S. Zmerli (Eds.), Political trust: Why context matters (pp. 13-46). Colchester: ECPR Press.

Marien, S. (2017). The measurement equivalence of political trust. In T. W. G. Van der Meer \& S. Zmerli (Eds.), Handbook of Political Trust. Cheltenham UK: Edward Elgar Publishing.

Mehta, P. D., \& Neale, M. C. (2005). People are variables too: Multilevel structural equations modeling. Psychological Methods, 10(3), 259.

Meredith, W. (1993). Measurement invariance, factor analysis and factorial invariance. Psychometrika, 58(4), 525-543. 
Meuleman, B., \& Billiet, J. (2009). A Monte Carlo sample size study: How many countries are needed for accurate multilevel SEM? Survey Research Methods, 3(1), 45-58.

Meuleman, B., Davidov, E., \& Billiet, J. (2009). Changing attitudes toward immigration in Europe, 2002-2007: A dynamic group conflict theory approach. Social Science Research, 38(2), 352-365.

Muthén, B. O. (1990). Mean and covariance structure analysis of hierarchical data. Department of Statistics, UCLA Statistics Series, No 62. Los Angeles: University of California.

Muthén, B. O. (1991). Multilevel factor analysis of class and student achievement components. Journal of Educational Measurement, 28(4), 338-354.

Muthén, B. O. (1994). Multilevel covariance structure analysis. Sociological Methods \& Research, 22(3), 376-398.

Muthén, B. O., \& Muthén, L. K. (2012). Mplus 7 base program. Los Angeles, CA: Muthén \& Muthén.

Muthén, B. O., \& Asparouhov, T. (2013). New methods for the study of measurement invariance with many groups. Unpublished technical report. http://www.statmodel.com. Accessed 14 June 2017.

Muthén, B. O., \& Satorra, A. (1995). Complex sample data in structural equation modeling. Sociological Methodology, 25, 267-316.

Muthén, L.K., \& Muthén, B.O. (2004). Mplus User's Guide, third ed. Muthén \& Muthén, Los Angeles, CA.

Oskarsson, S. (2010). Generalized trust and political support: A cross-national investigation. Acta Politica, 45(4), 423-443.

Poznyak, D., Meuleman, B., Abts, K., \& Bishop, G. F. (2014). Trust in American government: Longitudinal measurement equivalence in the ANES, 1964-2008. Social Indicators Research, 118(2), 741-758.

Puntscher, S., Hauser, C., Walde, J., \& Tappeiner, G. (2016). Measuring social capital with aggregated indicators: A case of ecological fallacy? Social Indicators Research, 125(2), 431-449.

Rabe-Hesketh, S., Skrondal, A., \& Pickles, A. (2004). Generalized multilevel structural equation modeling. Psychometrika, 69(2), 167-190.

Raudenbush, S. W., \& Bryk, A. S. (2002). Hierarchical linear models: Applications and data analysis methods. 2nd ed., SAGE, Thousand Oaks, CA.

Rothstein, B., \& Stolle, D. (2008). The state and social capital: An institutional theory of generalized trust. Comparative Politics, 40(4), 441-459.

Schneider, I. (2016). Can we trust measures of political trust? Assessing measurement equivalence in diverse regime types. Social Indicators Research, 133(3), 963-984.

Schwartz, S. H. (1992). Universals in the content and structure of values: Theoretical advances and empirical tests in 20 countries. Advances in Experimental Social Psychology, 25, 1-65.

Schwartz, S. H. (1994). Beyond individualism/collectivism: New cultural dimensions of values. In U. Kim, H. C. Triandis, C. Kagitcibasi, S. C. Choi, \& G. Yoon (Eds.), Individualism and collectivism: Theory, method and applications (pp. 85-119). SAGE.

Schwartz, S. H. (2006). A theory of cultural value orientations: Explication and applications. Comparative sociology, 5(2), 137-182. 
Schweig, J. (2014). Cross-level measurement invariance in school and classroom environment surveys: Implications for policy and practice. Educational Evaluation and Policy Analysis, 36(3), 259280.

Snijders, T., \& Bosker, R. (1999). Multilevel modeling: An introduction to basic and advanced multilevel modeling. SAGE, London.

Stankov, L., \& Saucier, G. (2015). Social axioms in 33 countries: Good replicability at the individual but less so at the country level. Journal of Cross-Cultural Psychology, 46(2), 296-315.

Steenkamp, J. B. E., \& Baumgartner, H. (1998). Assessing measurement invariance in cross-national consumer research. Journal of Consumer Research, 25(1), 78-90.

Stegmueller, D. (2013). How many countries for multilevel modeling? A comparison of frequentist and Bayesian approaches. American Journal of Political Science, 57(3), 748-761.

Tay, L., Woo, S. E., \& Vermunt, J. K. (2014). A conceptual and methodological framework for psychometric isomorphism: Validation of multilevel construct measures. Organizational Research Methods, 17(1), 77-106.

Turper, S., \& Aarts, K. (2017). Political Trust and Sophistication: Taking Measurement Seriously. Social Indicators Research, 130(1), 415-434.

Vandenberg, R. J., \& Lance, C. E. (2000). A review and synthesis of the measurement invariance literature: Suggestions, practices, and recommendations for organizational research. Organizational Research Methods, 3(1), 4-70.

van de Schoot, R., \& Depaoli, S. (2014). Bayesian analyses: Where to start and what to report. European Health Psychologist, 16(2), 75-84.

Van der Brug, W., \& Van Praag, P. (2007). Erosion of political trust in the Netherlands: Structural or temporarily? A research note. Acta Politica, 42(4), 443-458.

Van der Veld, W. M., \& Saris, W. E. (2011). Causes of generalized social trust. In E. Davidov, P. Schmidt \& J. Billiet (Eds.), Cross-cultural analysis: Methods and applications (pp. 207-247). New York, London: Routledge.

Van de Vijver, F. J. R. (1998). Towards a theory of bias and equivalence. Zuma Nachrichten Spezial, Cross-cultural survey equivalence, 3, 41-65.

Van de Vijver, F. J., \& Poortinga, Y. H. (2002). Structural equivalence in multilevel research. Journal of Cross-Cultural Psychology, 33(2), 141-156.

Van de Vijver, F. J. R., Van Hemert, D. A., \& Poortinga, Y. H. (Eds.). (2008). Individuals and cultures in multilevel analysis. Mahwah, NJ: Lawrence Erlbaum.

Van de Vijver, F. J., \& Watkins, D. (2006). Assessing similarity of meaning at the individual and country level. European Journal of Psychological Assessment, 22(2), 69-77.

Zmerli, S., \& Newton, K. (2008). Social trust and attitudes toward democracy. Public Opinion Quarterly, 72(4), 706-724.

Zyphur, M. J., Kaplan, S. A., \& Christian, M. S. (2008). Assumptions of cross-level measurement and structural invariance in the analysis of multilevel data: Problems and solutions. Group Dynamics: Theory, Research, and Practice, 12(2), 127. 